\title{
Hypersensitive C-reactive protein-albumin ratio predicts symptomatic intracranial hemorrhage after endovascular therapy in acute ischemic stroke patients
}

Qiang Peng ${ }^{1 \dagger}$, Jiankang Hou ${ }^{1 \dagger}$, Siyu Wang ${ }^{1 \dagger}$, Feng Zhou', Yan E ${ }^{1}$, Wei Wang ${ }^{1}$, Ting Huang ${ }^{1}$, Meng Wang ${ }^{1}$, Shi Huang ${ }^{1}$, Junshan Zhou ${ }^{1 *}$, Nihong Chen ${ }^{1,2^{*}}$ and Yingdong Zhang ${ }^{1 *}$

\begin{abstract}
Background: Approximately $10 \%$ of patients would develop symptomatic intracranial hemorrhage (sICH) after endovascular therapy. The aim of our study was to explore the ability of hypersensitive C-reactive protein-albumin ratio (HAR) in predicting $\mathrm{sICH}$ after endovascular therapy.

Methods: From April 2016 to December 2018, 334 consecutive patients with anterior circulation infarction undergoing endovascular therapy were enrolled in our study. sICH was defined using Heidelberg bleeding classification after endovascular therapy. Multiple regression analysis was used to investigate the potential risk factors of sICH after endovascular therapy. We used receiver operating characteristic curve analysis and nomogram analysis to assess the overall discriminative ability of the HAR in predicting $\mathrm{SICH}$ after endovascular therapy.

Results: Among these 334 patients enrolled, 37 (11.1\%) patients with anterior circulation infarction were identified with sICH after endovascular therapy. Univariate logistic regression analysis demonstrated that patients with higher levels of HAR may be inclined to develop sICH (odds ratio, 10.994; 95\% confidence interval, 4.567-26.463; $P=0.001$ ). This association remained significant even after adjustment for potential confounders. Also, a cutoff value of $0.526 \times$ $10^{-3}$ for HAR was detected in predicting sICH (area under curve, 0.763). Furthermore, nomogram analysis also suggested that HAR was an indicator of $\mathrm{sICH}$ (C-index was $0.890, P<0.001$ ).
\end{abstract}

Conclusions: This study showed that high levels of HAR could predict sICH after endovascular therapy.

Keywords: Hypersensitive C-reactive protein-albumin ratio, Acute ischemic stroke, Endovascular therapy, Symptomatic intracranial hemorrhage, Risk factor

\footnotetext{
*Correspondence: zhoujunshan6807@163.com; neon_2000@163.com; zhangyingdong@aliyun.com

${ }^{\dagger}$ Qiang Peng, Jiankang Hou and Siyu Wang contributed equally to this work. 'Department of Neurology, Nanjing First Hospital, Nanjing Medical University, No. 68 Changle Road, Nanjing 210006, P.R. China

Full list of author information is available at the end of the article
}

(c) The Author(s). 2021 Open Access This article is licensed under a Creative Commons Attribution 4.0 International License, which permits use, sharing, adaptation, distribution and reproduction in any medium or format, as long as you give appropriate credit to the original author(s) and the source, provide a link to the Creative Commons licence, and indicate if changes were made. The images or other third party material in this article are included in the article's Creative Commons licence, unless indicated otherwise in a credit line to the material. If material is not included in the article's Creative Commons licence and your intended use is not permitted by statutory regulation or exceeds the permitted use, you will need to obtain permission directly from the copyright holder. To view a copy of this licence, visit http://creativecommons.org/licenses/by/4.0/ The Creative Commons Public Domain Dedication waiver (http://creativecommons.org/publicdomain/zero/1.0/) applies to the data made available in this article, unless otherwise stated in a credit line to the data. 


\section{Background}

Stroke is one of leading causes of death and long-term disability globally [1]. According to several randomized controlled trials focusing on acute ischemic stroke because of large vessel occlusion in the anterior circulation, endovascular treatment has been demonstrated benefits in abating mortality and morbidity of stroke patients [2-6]. Nevertheless, some patients may develop symptomatic intracranial hemorrhage (sICH) within $24 \mathrm{~h}$ after endovascular therapy, which has been reported to be associated with symptoms worsening as well as poor outcomes [7, 8]. Therefore, it is notable for neurologists to explore the risk factors and predictors of $\mathrm{sICH}$ after endovascular therapy.

It is reported that inflammation may be related to central nervous system diseases, such as stroke, Parkinson's Disease and Alzheimer disease, and plays a crucial role in the mechanism [9-12]. Gong and his colleagues found the association between high-sensitivity C-reactive protein at admission and progressive motor deficits in patients with penetrating artery infarctions [13]. Previous studies also discovered that albumin might be able to attenuate excessive innate immunity in subarachnoid hemorrhage [14, 15]. High-sensitivity C-reactive protein to albumin ratio (HAR), a novel biomarker which combines high-sensitivity C-reactive protein and albumin, was reported to have to do with left ventricular thrombus formation following acute anterior myocardial infarction [16]. According to a recent study, increased serum HAR may be independently associated with the severity of carotid artery stenosis [17]. Moreover, the relationship between $\mathrm{C}$-reactive protein to albumin ratio and 90-day mortality in patients with acute ischemic stroke has been confirmed [18].

However, the correlation between HAR and sICH after endovascular treatment has not been clarified. Hence, this observational study was performed to explore the predictive utility of HAR in sICH after endovascular treatment, prospectively.

\section{Methods}

\section{Study population}

This prospective study was performed from April 2016 to December 2018 in Nanjing First Hospital, Nanjing Medical University. Acute ischemic stroke patients with anterior circulation infarction, who were treated with endovascular therapy within $6 \mathrm{~h}$ after symptom onset, were included in the study. The patients treated with a bridging therapy, which consists of intravenous thrombolysis and endovascular therapy, were also included. The exclusion criteria were as follows: (1) age less than 18 years; (2) posterior circulation infarction; (3) systemic inflammatory disease, renal failure, hepatic failure, brain tumor and presence of an active infection. Informed consent was obtained from participants or legal representatives, and the protocol was approved by the Ethical Committee of Nanjing First Hospital, Nanjing Medical University.

\section{Clinical assessments}

Clinical assessments were performed within $24 \mathrm{~h}$ after admission. All participants had standard assessments of demographic characteristics (including age and sex), vascular risk factors (including hypertension, diabetes mellitus, dyslipidemia, previous stroke, atrial fibrillation, coronary heart disease, current smoking and current drinking), previous antiplatelet, systolic blood pressure (SBP) at admission, diastolic blood pressure (DBP) at admission, time from door to puncture, intravenous thrombolysis, number of thrombectomy device passes, stroke severity, stroke subtype, and laboratory data. Computed tomography, magnetic resonance, digital subtraction angiography, electrocardiogram, transcranial Doppler and carotid ultrasonography were performed for assessing the stroke etiology. Stroke subtype was classified according to Trial of Org 10,172 in Acute Stroke Treatment (TOAST) criteria [19]. Successful recanalization was defined as a TICI score of 2b-3 [20]. Collateral status was assessed based on digital subtracted angiography using the American Society of Interventional and Therapeutic Neuroradiology/Society of Interventional Radiology grading system, with grade 0 to 1 representing poor collateral status and grade 2 to 4 representing moderate to excellent [21]. The blood samples to measure the levels of Hs-CRP and albumin were collected at $7 \mathrm{AM}$ the next day after admission. The measurement of Hs-CRP and albumin was completed within $24 \mathrm{~h}$ after admission.

\section{Definition of $\mathrm{sICH}$}

Symptomatic Intracranial Hemorrhage ( $\mathrm{sICH}$ ) was defined as any hemorrhagic transformation associated with total NIHSS score worsening $\geq 4$ points or worsening $\geq 2$ points in one NIHSS category or deterioration led to intubation, hemicraniectomy, external ventricular drain placement, or any other major interventions, which used the Heidelberg bleeding classification [7, 22, 23]. The evaluation of neurological deficits was conducted using National Institutes of Health Stroke Scale (NIHSS) score on admission and continued the following $24 \mathrm{~h}$ after endovascular therapy by two certified neurologists blind to clinical data. In case of disagreement about the NIHS $\mathrm{S}$ score evaluation, a third neurologist was invited for a final decision.

\section{Measurement of laboratory data}

All the blood samples were collected at 7 AM the next day after admission. The levels of Hs-CRP were measured with an immunoturbidimetry assay by an Architect c16000 chemistry analyzer (Abbott Diagnostics, 
Chicago, USA). The levels of albumin were measured with a chemiluminescence detection assay by an AU5800 chemistry analyzer (Beckman Coulter, Brea, USA).

\section{Statistical analysis}

Statistical analyses were performed with $\mathrm{R}$ version 3.6.2 software (http://www.R-project.org/). Categorical variables were expressed as $\mathrm{n}(\%)$ and continuous variables were expressed as means (standard deviation, SD) or medians (interquartile range, IQR). Differences in baseline characteristics between groups were analyzed using independent sample t-tests or Mann-Whitney $U$ tests for continuous variables as well as the Chi-squared test or Fisher's exact test for categorical variables, as appropriate. Multivariable analysis was adjusted for all potential confounders with statistically significant association at $P<0.05$ in univariate regression analysis. Receiver operating characteristic (ROC) curve analysis was performed to assess the overall discriminative ability of the HAR to predict sICH after endovascular therapy and to establish optimal cutoff points at which the sum of the specificity and sensitivity was the highest. A MedCalc 15.6.0 (MedCalc Software Acacialaan 22, B-8400 Ostend, Belgium) packet program was used to obtain ROC. A nomogram based on the independent predictors was constructed by $\mathrm{R}$ software with the package rms. The predictive capacity of the nomogram was determined by Harrell's c-index. A two-tailed value of $P<0.05$ was considered significant.

\section{Results}

From April 2016 to December 2018, five hundred and twelve patients were enrolled in this study. One hundred and seventy-eight patients were excluded for the reasons below: posterior circulation infarct $(n=149)$, systemic inflammatory disease $(n=3)$, renal failure $(n=16)$, hepatic failure $(n=4)$, brain tumor $(n=1)$ and presence of an active infection $(n=5)$. A total of 334 subjects $(210$ men; mean age, $69.0 \pm 11.2$ years) were included for the final analysis. Among these patients, 229 (68.6\%) had hypertension, 67 (20.1\%) had diabetes mellitus, 100 (29.9\%) had dyslipidemia, 75 (22.5\%) had coronary heart disease, and 99 (29.6\%) had atrial fibrillation.

Among the 334 patients enrolled, sICH was observed in 37 patients (11.1\%). Table 1 showed the comparisons of baseline characteristics in patients with or without sICH after endovascular therapy. Compared to the patients without $\mathrm{sICH}$ after endovascular therapy, those patients with sICH were older $(P=0.016)$ and had higher proportions of hypertension $(P=0.014)$ as well as previous antiplatelet use $(P=0.003)$; Higher levels of SBP $(P=0.017)$, initial NIHSS score $(P=0.043)$, the number of thrombectomy device passes $(P=0.048)$, fasting blood glucose $(P=0.001)$ and $\operatorname{HAR}(P=0.001)$.

Table 2 showed the results of logistic regression analysis for risk factors with $\mathrm{sICH}$ after endovascular therapy. Univariate logistic regression analysis exhibited that HAR [odds ratio (OR), 10.994; 95\% confidence interval (CI) 4.567-26.463, $P=0.001$ ], age (OR, $1.044 ; 95 \% \mathrm{CI}$ 1.008-1.081, $P=0.017$ ), hypertension (OR, 3.249; 95\% CI $1.228-8.594, P=0.018)$, previous antiplatelet use (OR, 3.225; 95\% CI 1.548-6.718, $P=0.002$ ), SBP (OR, 1.017; 95\% CI 1.003-1.031, $P=0.019)$, initial NIHSS score (OR, 1.058 ; $95 \%$ CI $1.011-1.107, P=0.014)$, the number of thrombectomy device passes (OR, 1.362; 95\% CI 1.0461.722, $P=0.022)$ and fasting blood glucose level (OR, 1.273 ; $95 \%$ CI $1.140-1.421, P=0.001)$ might be associated with sICH after endovascular therapy. After adjustment for all potential confounders, HAR was identified as an independent risk factor for sICH after endovascular therapy (OR, 12.384; 95\% CI 4.379-35.023, $P=$ 0.001). Moreover, fasting blood glucose level (OR, 1.333; 95\% CI 1.145-1.552, $P=0.001)$ and previous antiplatelet use (OR, 4.940; 95\% CI 1.920-12.713, $P=0.001)$ were also related to $\mathrm{SICH}$, independently.

Figure 1 exhibited the ROC curve, which showed the predictive ability of HAR in sICH after endovascular therapy. The optimal cutoff value for HAR as a predictor of $\mathrm{sICH}$ was determined as $0.526 \times 10^{-3}$ in the ROC curve analysis, which yielded a sensitivity of $54.1 \%$ and a specificity of $88.6 \%$, with the AUC at $0.763(95 \% \mathrm{CI}$, 0.714-0.808). What is more, we also compared the ROC of HAR and Hs-CRP. The AUCs of HAR and Hs-CRP are 0.763 and 0.718 , respectively. According to $\mathrm{Z}$ test about pairwise comparison of ROC curves, HAR is able to predict better than Hs-CRP alone $(P=0.0003)$, which can be seen in the Supplementary Figure 1 and Supplementary Figure 2.

Figure 2 showed the nomogram. The concordance index was $0.890(P<0.001)$. The novel model indicated that higher HAR was an independent indicator of $\mathrm{sICH}$ after endovascular therapy. These findings were similar to those findings above, which was obtained previously in the multivariate logistic model and ROC curve analysis.

\section{Discussion}

This observational research helped us find the relationship between HAR and sICH after endovascular treatment. The anterior circulation infarction patients undergoing endovascular treatment with elevated levels of HAR are prone to develop sICH. After the adjustment for fasting blood glucose level, previous antiplatelet use and some other variables, HAR remains a significant independent risk factor of sICH after endovascular treatment. It is widely believed that one biomarker with $0.7<$ 
Table 1 Characteristics of subgroups based on the presence of $\mathrm{slCH}$

\begin{tabular}{|c|c|c|c|}
\hline Variable & sICH group $(n=37)$ & Non-sICH group $(n=297)$ & $P$ \\
\hline \multicolumn{4}{|l|}{ Demographic characteristics } \\
\hline Age, years & $73.2 \pm 10.9$ & $68.5 \pm 11.2$ & 0.016 \\
\hline Male, \% & $23(62.2)$ & $187(63.0)$ & 0.904 \\
\hline \multicolumn{4}{|l|}{ Vascular risk factors, \% } \\
\hline Hypertension & $32(86.5)$ & $197(66.3)$ & 0.014 \\
\hline Diabetes mellitus & $9(24.3)$ & $58(19.5)$ & 0.515 \\
\hline Dyslipidemia & $7(18.9)$ & $93(31.3)$ & 0.132 \\
\hline Coronary heart disease & $12(32.4)$ & $63(21.2)$ & 0.529 \\
\hline Atrial fibrillation & $14(37.8)$ & 85 (28.6) & 0.076 \\
\hline Previous stroke & $9(24.3)$ & $70(23.6)$ & 0.100 \\
\hline Current drinking & $8(21.6)$ & $82(27.6)$ & 0.421 \\
\hline Current smoking & $9(24.3)$ & $102(34.3)$ & 0.144 \\
\hline \multicolumn{4}{|l|}{ Clinical data } \\
\hline Previous antiplatelet, \% & $14(37.8)$ & $47(15.8)$ & 0.003 \\
\hline $\mathrm{SBP}, \mathrm{mmHg}$ & $149.7 \pm 22.7$ & $139.8 \pm 23.9$ & 0.017 \\
\hline $\mathrm{DBP}, \mathrm{mmHg}$ & $84.1 \pm 17.7$ & $85.2 \pm 14.6$ & 0.668 \\
\hline Body mass index, $\mathrm{kg} / \mathrm{m}^{2}$ & $24.2 \pm 3.8$ & $24.1 \pm 3.7$ & 0.919 \\
\hline Initial NIHSS, score & $16(13,22)$ & $15(11,19)$ & 0.043 \\
\hline Intravenous thrombolysis, \% & $21(56.8)$ & $122(41.1)$ & 0.069 \\
\hline Time from door to puncture, min & $120.0(90.5,149.0)$ & $110.0(75.0,140.0)$ & 0.075 \\
\hline Number of thrombectomy device passes & $2(1,3)$ & $2(1,3)$ & 0.048 \\
\hline Poor collateral status, \% & $23(62.2)$ & $135(45.5)$ & 0.055 \\
\hline Successful recanalization, \% & $33(89.2)$ & $256(86.2)$ & 0.800 \\
\hline Stroke subtype, \% & & & 0.169 \\
\hline LAA & $12(32.4)$ & $139(46.8)$ & \\
\hline CE & $21(56.8)$ & $121(40.7)$ & \\
\hline Other subtype & $4(10.8)$ & $37(12.5)$ & \\
\hline \multicolumn{4}{|l|}{ Laboratory data } \\
\hline $\mathrm{TC}, \mathrm{mmol} / \mathrm{L}$ & $4.2 \pm 1.2$ & $4.4 \pm 1.2$ & 0.537 \\
\hline $\mathrm{TG}, \mathrm{mmol} / \mathrm{L}$ & $1.1(0.8,1.7)$ & $1.1(0.8,1.6)$ & 0.375 \\
\hline $\mathrm{LDL}, \mathrm{mmol} / \mathrm{L}$ & $2.6(1.9,3.2)$ & $2.6(2.0,3.2)$ & 0.469 \\
\hline $\mathrm{HDL}, \mathrm{mmol} / \mathrm{L}$ & $1.1 \pm 0.3$ & $1.1 \pm 0.4$ & 0.865 \\
\hline $\mathrm{FBG}, \mathrm{mmol} / \mathrm{L}$ & $9.0 \pm 3.5$ & $6.9 \pm 2.4$ & 0.001 \\
\hline Homocysteine, $\mu \mathrm{mol} / \mathrm{L}$ & $12.8(10.7,16.0)$ & $12.3(10.4,15.8)$ & 0.588 \\
\hline HAR, $10^{-3}$ & $0.54(0.24,1.55)$ & $0.17(0.09,0.33)$ & 0.001 \\
\hline
\end{tabular}

Abbreviation: NIHSS, National institute of health stroke scale, SBP Systolic blood pressure, DBP Diastolic blood pressure, LAA Large-artery atherosclerosis, CE Cardioembolism, TC Total cholesterol, TG Triglyceride, LDL Low-density lipoprotein, HDL High-density lipoprotein, FBG Fasting blood glucose, HAR Hypersensitive C-reactive protein-albumin ratio

area under the curve $<0.9$ presents a moderate diagnostic value $[9,24]$. Therefore, HAR might serve as a biomarker associated with sICH after endovascular treatment. Furthermore, the nomogram about $\mathrm{sICH}$ after endovascular treatment confirmed this conclusion.

In our study, the incidence of sICH after endovascular treatment is $11.1 \%$, which is consistent with previous studies [23, 25]. This may be relevant to the rigorous evaluation in our stroke center. HAR has been reported to be related to the severity of carotid artery stenosis, which is related to the severity and prognosis of stroke [17]. A recent study showed that high levels of HAR at admission were significantly associated with intrahospital mortality in the patients with intracerebral hemorrhage, another type of stroke [26]. Kocatürk M and colleagues also found the relationship between HAR 
Table 2 Logistic regression analysis for risk factors with symptomatic intracranial hemorrhage after endovascular therapy

\begin{tabular}{|c|c|c|c|c|}
\hline Variable & Unadjusted OR (95\%Cl) & $P$ & Adjusted OR $(95 \% \mathrm{Cl})$ & $P$ \\
\hline \multicolumn{5}{|l|}{ Demographic characteristics } \\
\hline Age, years & $1.044(1.008-1.081)$ & 0.017 & $1.028(0.984-1.074)$ & 0.215 \\
\hline Male & $0.958(0.473-1.938)$ & 0.904 & & \\
\hline \multicolumn{5}{|l|}{ Vascular risk factors } \\
\hline Hypertension & $3.249(1.228-8.594)$ & 0.018 & $3.478(0.956-12.657)$ & 0.059 \\
\hline Diabetes mellitus & $1.325(0.593-2.959)$ & 0.493 & & \\
\hline Dyslipidemia & $0.512(0.217-1.208)$ & 0.126 & & \\
\hline Coronary heart disease & $1.783(0.849-3.746)$ & 0.127 & & \\
\hline Atrial fibrillation & $1.518(0.746-3.089)$ & 0.249 & & \\
\hline Previous stroke & $1.038(0.467-2.304)$ & 0.927 & & \\
\hline Current drinking & $0.720(0.316-1.640)$ & 0.434 & & \\
\hline Current smoking & $0.661(0.278-1.345)$ & 0.221 & & \\
\hline \multicolumn{5}{|l|}{ Clinical data } \\
\hline Previous antiplatelet & $3.225(1.548-6.718)$ & 0.002 & $4.940(1.920-12.713)$ & 0.001 \\
\hline SBP & $1.017(1.003-1.031)$ & 0.019 & $1.012(0.995-1.030)$ & 0.174 \\
\hline DBP & $0.995(0.972-1.018)$ & 0.667 & & \\
\hline Body mass index & $1.005(0.916-1.102)$ & 0.919 & & \\
\hline Initial NIHSS & $1.058(1.011-1.107)$ & 0.014 & $1.033(0.970-1.101)$ & 0.306 \\
\hline Intravenous thrombolysis & $1.883(0.944-3.755)$ & 0.072 & & \\
\hline Time from door to puncture & $1.002(0.998-1.006)$ & 0.329 & & \\
\hline Number of thrombectomy device passes & $1.362(1.046-1.772)$ & 0.022 & $1.396(0.982-1.985)$ & 0.063 \\
\hline Poor collateral status & $1.971(0.976-3.980)$ & 0.058 & & \\
\hline Successful recanalization & $1.321(0.445-3.925)$ & 0.616 & & \\
\hline \multicolumn{5}{|l|}{ Stroke subtype, \% } \\
\hline LAA & Reference & & & \\
\hline CE & $0.799(0.243-2.620)$ & 0.711 & & \\
\hline Other subtype & $1.605(0.518-4.974)$ & 0.412 & & \\
\hline \multicolumn{5}{|l|}{ Laboratory data } \\
\hline $\mathrm{TC}$ & $0.906(0.663-1.238)$ & 0.535 & & \\
\hline TG & $1.129(0.831-1.532)$ & 0.438 & & \\
\hline $\mathrm{HDL}$ & $0.917(0.338-2.487)$ & 0.865 & & \\
\hline LDL & $0.906(0.621-1.322)$ & 0.609 & & \\
\hline FBG & $1.273(1.140-1.421)$ & 0.001 & $1.333(1.145-1.552)$ & 0.001 \\
\hline Homocysteine & $1.000(0.999-1.001)$ & 0.865 & & \\
\hline HAR & $10.994(4.567-26.463)$ & 0.001 & $12.384(4.379-35.023)$ & 0.001 \\
\hline
\end{tabular}

Abbreviation: NIHSS, National institute of health stroke scale, SBP Systolic blood pressure, DBP Diastolic blood pressure, LAA Large-artery atherosclerosis, CE Cardioembolism, TC Total cholesterol, TG Triglyceride, LDL Low-density lipoprotein, HDL High-density lipoprotein, FBG Fasting blood glucose, HAR Hypersensitive C-reactive protein-albumin ratio

and the prognosis in patients with acute stroke [18]. To our knowledge, this study is the first to investigate the prospective association of HAR with sICH after endovascular treatment, and reveals that HAR could act as a useful predictor for $\mathrm{sICH}$ in patients undergoing endovascular treatment, according to the results of multivariate logistic regression, ROC analysis as well as nomogram analysis. Our study also found that previous antiplatelet use and fasting blood glucose level are associated with $\mathrm{sICH}$ after endovascular treatment, which is in line with other researches $[25,27,28]$.

The mechanisms underlying the association between HAR and sICH after endovascular treatment are not entirely clear, and further studies are warranted. In term of previous researches, inflammation plays an important role in the pathophysiological mechanism of ischemic 


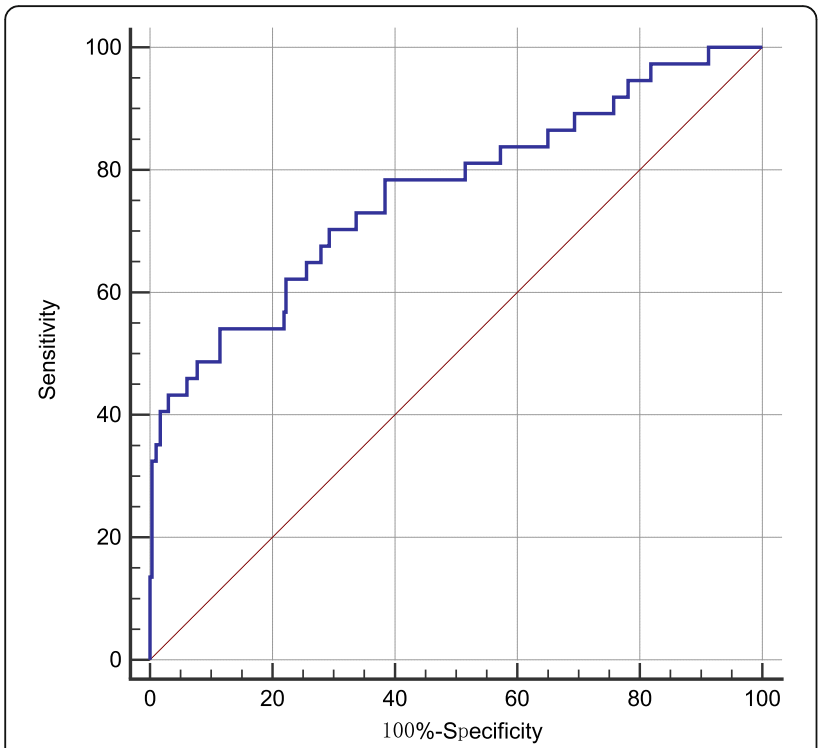

Fig. 1 Receiver operating characteristic (ROC) curve for the value of HAR to predict sICH

stroke [29-32]. Oxidative stress and inflammatory activity could lead to blood brain barrier disruption, which may be linked to hemorrhagic transformation after endovascular treatment [33]. High sensitivity C-reactive protein is a crucial inflammatory marker. In response to IL-1, IL- 6 and TNF- $\alpha$, hepatocytes produced high sensitivity C-reactive protein in large amounts [34, 35]. Proinflammatory cytokine, might be involved in the pathophysiology of sICH after endovascular treatment through inflammatory responses, may be induced by high sensitivity C-reactive protein. In addition, several clinical studies also found that high-sensitivity Creactive protein is associated with progressive stroke [9] and post-stroke fatigue [36]. Moreover, it is believed that albumin, a heart-shaped plasma protein with a 585 amino acid polypeptide chain, possesses neuroprotective effect, and serum albumin levels are likely lower in inflammatory states [37]. Albumin might serve as an antiinflammatory agent and attenuate microglial and $\mathrm{T}$ cell activation [38]. Albumin administration has been proven to improve neurobehavior in experimental subarachnoid hemorrhage [15]. Nevertheless, only single biomarker might be affected by pathophysiological changes. HAR, the combination of high sensitivity $\mathrm{C}$-reactive protein and albumin, may be able to provide more compound information in predicting $\mathrm{sICH}$ in patients undergoing endovascular treatment.

Although we have discovered the relationship between HAR and sICH after endovascular treatment, there are still some limitations in this clinical research. First, this study is an observational research, not randomized controlled trial. The sample size of our observational research is also relatively small. Second, this research was conducted in a single stroke center in China, so the conclusion is supposed to be verified in other stroke center to confirm that this finding can be extrapolated to other populations. Third, it is reported that several biomarkers may change during hospitalization [39]. Therefore, in

\begin{tabular}{|c|c|c|c|c|c|c|c|c|c|c|c|c|c|c|c|}
\hline & 0 & & 10 & & 20 & 30 & 40 & & & 60 & 70 & 80 & & 90 & 100 \\
\hline HAR & 0 & & 0.5 & & 1 & 1.5 & & 2 & 2.5 & & 3 & 3.5 & & 4 & 4.5 \\
\hline FBG & 0 & 2 & 4 & 6 & 10 & 12 & 16 & $\sqrt{18}$ & & & & & & & \\
\hline Previous.antiplatelet & 0 & 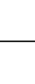 & 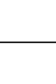 & 4 & & & & & & & & & & & \\
\hline Total Points & 0 & & 0 & 20 & 30 & 40 & 50 & 60 & 70 & 80 & 90 & 100 & 110 & 120 & 130 \\
\hline Risk & & & 0.01 & & 0.1 & 0.3 & 0.5 & 0.7 & 0.9 & & $\overrightarrow{0.99}$ & & & & \\
\hline
\end{tabular}


further studies, we should examine the biomarker, such as high sensitivity $\mathrm{C}$-reactive protein and albumin, dynamically. What is more, we do not have accurate data about the duration of the endovascular procedure, but we aim to evaluate them in our further studies to explore the relationship between the duration of the endovascular procedure and $\mathrm{sICH}$ after endovascular treatment. Despite the shortcomings above, this study has found the predictive value of HAR for $\mathrm{sICH}$ after endovascular treatment, which is helpful for exploring the mechanism of sICH after endovascular treatment.

\section{Conclusion}

In summary, the results of our study indicated that the higher levels of HAR were associated with increasing risk of sICH after endovascular treatment. Moreover, HAR may be able to serve as a non-invasive predictor for $\mathrm{sICH}$ in patients with stroke undergoing endovascular treatment. More multicenter studies with large sample size are needed in the future. In addition, neurologists may pay more attention to the patients with higher levels of HAR after endovascular treatment in the future clinical practice.

\section{Supplementary Information}

The online version contains supplementary material available at https://doi. org/10.1186/s12883-021-02066-2.

Additional file 1. The comparison of $\mathrm{ROC}$ curves for $\mathrm{sICH}$ after endovascular therapy.

Additional file 2. The details about the comparison of ROC curves for sICH after endovascular therapy.

\section{Acknowledgments}

We express gratitude to all the patients who participated in this study.

\section{Authors' contributions}

QP, JH and SW for formal analysis and writing - original draft; FZ, YE, WW, TH, MW and SH for data curation; JZ, NC and YZ for writing - review \& editing and conceptualization. The authors read and approved the final manuscript.

\section{Funding}

This work was supported by Nanjing Medical Science and Technology Development Foundation (ZDX16002, ZKX16050 and YKK18097) and Natural Science Foundation of Jiangsu Province (BK20201117). These fundings play role in the collection and analysis of data.

\section{Availability of data and materials}

The data that support the findings of this study are available from the corresponding author upon reasonable request.

\section{Ethics approval and consent to participate}

Informed consent was obtained from participants or legal representatives, and the protocol was approved by the Ethical Committee of Nanjing First Hospital, Nanjing Medical University.

\section{Consent for publication}

Not Applicable.

\section{Competing interests}

All the authors declare that there is no conflict of interest.

\section{Author details}

'Department of Neurology, Nanjing First Hospital, Nanjing Medical University, No. 68 Changle Road, Nanjing 210006, P.R. China. ²Department of Neurology, Nanjing Yuhua Hospital, Nanjing First Hospital, Nanjing Medical University,

Nanjing 210006, Jiangsu, China.

Received: 10 October 2020 Accepted: 20 January 2021

Published online: 01 February 2021

\section{References}

1. Powers WJ, Rabinstein AA, Ackerson T, et al. 2018 guidelines for the early Management of Patients with acute ischemic stroke: a guideline for healthcare professionals from the American Heart Association/American Stroke Association. Stroke. 2018;49(3):e46-e110.

2. Campbell BC, Mitchell PJ, Kleinig TJ, et al. Endovascular therapy for ischemic stroke with perfusion-imaging selection. N Engl J Med. 2015;372(11):1009-18.

3. Goyal M, Demchuk AM, Menon BK, et al. Randomized assessment of rapid endovascular treatment of ischemic stroke. N Engl J Med. 2015;372(11): 1019-30.

4. Berkhemer OA, Fransen PS, Beumer D, et al. A randomized trial of intraarterial treatment for acute ischemic stroke. N Engl J Med. 2015;372(1): $11-20$.

5. Saver JL, Goyal M, Bonafe A, et al. Stent-retriever thrombectomy after intravenous t-PA vs. t-PA alone in stroke. N Engl J Med. 2015;372(24):228595.

6. Jovin TG, Chamorro A, Cobo E, et al. Thrombectomy within 8 hours after symptom onset in ischemic stroke. N Engl J Med. 2015;372(24):2296-306.

7. Zhang $\mathrm{X}$, Yuan $\mathrm{K}$, Wang $\mathrm{H}$, et al. Nomogram to predict mortality of endovascular Thrombectomy for ischemic stroke despite successful recanalization. J Am Heart Assoc. 2020;9(3):e014899.

8. Manno C, Disanto G, Bianco G, et al. Outcome of endovascular therapy in stroke with large vessel occlusion and mild symptoms. Neurology. 2019; 93(17):e1618-26.

9. Gong P, Liu Y, Huang T, et al. The association between high-sensitivity Creactive protein at admission and progressive motor deficits in patients with penetrating artery infarctions. BMC Neurol. 2019;19(1):346.

10. Jiang T, Gong PY, Tan MS, et al. Soluble TREM1 concentrations are increased and positively correlated with total tau levels in the plasma of patients with Alzheimer's disease. Aging Clin Exp Res. 2019;31(12):1801-5.

11. Pajares M, I Rojo A, Manda G, Boscá L, Cuadrado A. Inflammation in Parkinson's disease: mechanisms and therapeutic implications. Cells. 2020; 9(7):E1687.

12. Mészáros Á, Molnár K, Nógrádi B, et al. Neurovascular Inflammaging in health and disease. Cells. 2020:9(7):E1614.

13. Gong $P$, Zhang X, Gong Y, et al. A novel nomogram to predict early neurological deterioration in patients with acute ischaemic stroke. Eur J Neurol. 2020;27(10):1996-2005

14. Xie $Y$, Liu W, Zhang $X$, et al. Human albumin improves long-term behavioral Sequelae after subarachnoid hemorrhage through neurovascular remodeling. Crit Care Med. 2015:43(10):e440-9.

15. Xie $Y$, Guo $H$, Wang $L$, et al. Human albumin attenuates excessive innate immunity via inhibition of microglial Mincle/Syk signaling in subarachnoid hemorrhage. Brain Behav Immun. 2017;60:346-60.

16. Cirakoglu OF, Aslan AO, Yilmaz AS, Şahin $S$, Akyüz AR. Association between C-reactive protein to albumin ratio and left ventricular thrombus formation following acute anterior myocardial infarction. Angiology. 2020;71:804

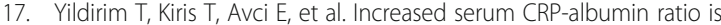
independently associated with severity of carotid artery stenosis. Angiology. 2020;71(8):740-6.

18. Kocatürk M, Kocatürk Ö. Assessment of relationship between C-reactive protein to albumin ratio and 90-day mortality in patients with acute ischaemic stroke. Neurologia I Neurochirurgia Pols. 2019;53(3):205-11.

19. Adams $\mathrm{H} J \mathrm{~J}$, Bendixen $B$, Kappelle $L$, et al. Classification of subtype of acute ischemic stroke: definitions for use in a multicenter clinical trial. TOAST. Trial of org 10172 in acute stroke treatment. Stroke. 1993;24:35-41.

20. Fernández Menéndez S, Murias Quintana E, Vega Valdés $P$, et al. Efficacy and safety of endovascular treatment in acute tandem carotid occlusions: analysis of a single-center cohort. Cerebrovasc Dis Extra. 2020;10(2):50-8

21. Zaidat $\mathrm{OO}$, Yoo AJ, Khatri $P$, et al. Recommendations on angiographic revascularization grading standards for acute ischemic stroke: a consensus statement. Stroke. 2013:44(9):2650-63. 
22. von Kummer R, Broderick JP, Campbell BC, et al. The Heidelberg bleeding classification: classification of bleeding events after ischemic stroke and reperfusion therapy. Stroke. 2015;46(10):2981-6.

23. Hao $\mathrm{Y}$, Yang $\mathrm{D}$, Wang $\mathrm{H}$, et al. Predictors for symptomatic intracranial hemorrhage after endovascular treatment of acute ischemic stroke. Stroke. 2017:48(5):1203-9.

24. Wu C, Li C, Zhao W, et al. Elevated trimethylamine $\mathrm{N}$-oxide related to ischemic brain lesions after carotid artery stenting. Neurology. 2018;90(15): e1283-90.

25. Li F, Ren Y, Cui $X$, et al. Postoperative hyperglycemia predicts symptomatic intracranial hemorrhage after endovascular treatment in patients with acute anterior circulation large artery occlusion. J Neurol Sci. 2020;409:116588.

26. Bender M, Haferkorn K, Friedrich M, Uhl E, Stein M. Impact of early Creactive protein/albumin ratio on intra-hospital mortality among patients with spontaneous Intracerebral hemorrhage. J Clin Med. 2020:9(4):1236.

27. Montalvo M, Mistry E, Chang AD, et al. Predicting symptomatic intracranial haemorrhage after mechanical thrombectomy: the TAG score. J Neurol Neurosurg Psychiatry. 2019;90(12):1370-4.

28. Merlino G, Sponza M, Gigli GL, et al. Prior use of antiplatelet therapy and outcomes after endovascular therapy in acute ischemic stroke due to large vessel occlusion: a single-center experience. J Clin Med. 2018;7(12):518.

29. Dhanesha N, Jain M, Tripathi AK, et al. Targeting myeloid-specific integrin a9 $\beta 1$ improves short- and long-term stroke outcomes in murine models with preexisting comorbidities by limiting thrombosis and inflammation. Circ Res. 2020;126(12):1779-94.

30. Parikh NS, Merkler AE, ladecola C. Inflammation, autoimmunity, infection, and stroke: epidemiology and lessons from therapeutic intervention. Stroke. 2020:51(3):711-8.

31. Gong P, Xie Y, Jiang T, et al. Neutrophil-lymphocyte ratio predicts postthrombolysis early neurological deterioration in acute ischemic stroke patients. Brain Behav. 2019;9(10):e01426.

32. Stoll G, Nieswandt B. Thrombo-inflammation in acute ischaemic stroke implications for treatment. Nat Rev Neurol. 2019:15(8):473-81.

33. Paciaroni $\mathrm{M}$, Agnelli $\mathrm{G}$, Caso V, et al. Acute hyperglycemia and early hemorrhagic transformation in ischemic stroke. Cerebrovasc Dis. 2009;28(2): $119-23$.

34. Butterweck V, Prinz S, Schwaninger M. The role of interleukin-6 in stressinduced hyperthermia and emotional behaviour in mice. Behav Brain Res. 2003;144(1-2):49-56

35. Lakhan SE, Kirchgessner A, Hofer M. Inflammatory mechanisms in ischemic stroke: therapeutic approaches. J Transl Med. 2009;7:97.

36. Liu X, Wang B, Wang X, Tian KM, Wang X, Zhang Y. Elevated plasma highsensitivity C-reactive protein at admission predicts the occurrence of poststroke fatigue at six months after ischemic stroke. Eur J Neurol. 2020;27: 2022. https://doi.org/10.1111/ene.14430

37. Anraku M, Shintomo R, Taguchi $\mathrm{K}$, et al. Amino acids of importance for the antioxidant activity of human serum albumin as revealed by recombinant mutants and genetic variants. Life Sci. 2015;134:36-41.

38. Ezra A, Rabinovich-Nikitin I, Rabinovich-Toidman P, Solomon B. Multifunctional effect of human serum albumin reduces Alzheimer's disease related pathologies in the 3xTg mouse model. J Alzheimers Dis. 2016;50(1): $175-88$.

39. Iadecola C, Anrather J. The immunology of stroke: from mechanisms to translation. Nat Med. 2011;17(7):796-808.

\section{Publisher's Note}

Springer Nature remains neutral with regard to jurisdictional claims in published maps and institutional affiliations.

\section{Ready to submit your research? Choose BMC and benefit from:}

- fast, convenient online submission

- thorough peer review by experienced researchers in your field

- rapid publication on acceptance

- support for research data, including large and complex data types

- gold Open Access which fosters wider collaboration and increased citations

- maximum visibility for your research: over $100 \mathrm{M}$ website views per year

At $\mathrm{BMC}$, research is always in progress.

Learn more biomedcentral.com/submissions 